\title{
Magnetic resonance imaging study of alteration of tibiofemoral joint articulation after posterior cruciate ligament injury
}

\author{
Sivashankar Chandrasekaran ${ }^{\text {a,c,* }}$, Jennifer M. Scarvell ${ }^{\text {a,c }}$, Graham Buirski ${ }^{\text {b,c }}$, Kevin R. Woods a , Paul N. Smith ${ }^{\text {a,c }}$ \\ a Trauma and Orthopaedic Research Unit, Canberra Hospital, PO Box 11, Woden ACT 2606 Australia \\ ${ }^{b}$ Department of Radiology, Canberra Hospital, Canberra, Australia \\ c Australian National University Medical School, Australia
}

\section{A R T I C L E I N F O}

\section{Article history:}

Received 14 June 2010

Received in revised form 17 November 2010

Accepted 27 November 2010

Available online xxxx

\section{Keywords:}

Posterior cruciate ligament

Posterior cruciate ligament injury

Tibiofemoral joint sagittal articulation

\begin{abstract}
A B S T R A C T
Cadaveric studies have shown that the posterior cruciate ligament (PCL) is an important constraint to posterior translation of the tibia. Arthroscopic studies have shown that chronic PCL injuries predispose to articular cartilage lesions in the medial compartment and the patellofemoral joint. The aim of the present study was to investigate sagittal plane articulation of the tibiofemoral joint of subjects with an isolated PCL injury.

Magnetic resonance was used to generate sagittal images of 10 healthy knees and 10 knees with isolated PCL injuries. The subjects performed a supine leg press against a $150 \mathrm{~N}$ load. Images were generated at $15^{\circ}$ intervals as the knee flexed from 0 to $90^{\circ}$. The tibiofemoral contact and the flexion facet centre (FFC) were measured from the posterior tibial cortex.

The contact pattern and FFC was significantly more anterior in the injured knee from 45 to $90^{\circ}$ of knee flexion in the medial compartment compared to the healthy knee. The greatest difference between the mean TFC points of both groups occurred at 75 and $90^{\circ}$, the difference being $4 \mathrm{~mm}$ and $5 \mathrm{~mm}$ respectively. The greatest difference between the mean FFC of both groups occurred at $75^{\circ}$ of flexion, which was $3 \mathrm{~mm}$. There was no significant difference in the contact pattern and FFC between the injured and healthy knees in the lateral compartment. Our findings show that there is a significant difference in the medial compartment sagittal plane articulation of the tibiofemoral joint in subjects with an isolated PCL injury.
\end{abstract}

Crown Copyright @ 2010 Published by Elsevier B.V. All rights reserved.

\section{Introduction}

Biomechanical studies show that the posterior cruciate ligament (PCL) is the most important constraint to posterior translation of the tibia above $30^{\circ}$ of knee flexion [1,2]. At flexion angles below this, the posterolateral complex is the most important restraint to posterior tibial translation [3]. The PCL is most commonly injured when a direct blow to the flexed knee pushes the tibia posteriorly relative to the femur [4]. In the literature, there is no clear consensus on the indications and benefits of surgical reconstruction of an injured PCL. PCL injuries are most often managed non-operatively with physiotherapy. Following non-operative management only a minority of subjects have persistent instability symptoms but in the elite sporting population the majority of subjects are unable to compete at preinjury level [5]. Recent arthroscopic studies have suggested that PCL injury does not have a benign natural history but may predispose to articular cartilage damage $[6,7]$. In one study,

\footnotetext{
* Corresponding author. Australian National University Medical School, Australia. Tel.: + 61411250466

E-mail addresses: dr_sivashankar@yahoo.com.au (S. Chandrasekaran), jennie.scarvell@act.gov.au (J.M. Scarvell), graham.buirski@act.gov.au (G. Buirski), kwoods@co.net.au (K.R. Woods), smithadmin@co.net.au (P.N. Smith).
}

arthroscopic evaluation of 181 subjects with a 5 year history of an isolated PCL injury, $80 \%$ of subjects had articular cartilage damage to the medial femoral condyle and $50 \%$ to the patella [8]. The aim of this study is to use magnetic resonance imaging (MRI) to determine the effects of PCL injury on the sagittal plane articulation of the tibiofemoral joint. The hypothesis of the study is that PCL injury would alter the sagittal plane articulation of the tibiofemoral joint.

\section{Methods}

\subsection{Study design}

This is a case control study that uses a MRI model, previously described to study ACL injuries [9-12], to study the effects of PCL injury on sagittal plane articulation of the tibiofemoral joint.

\subsection{Subject selection}

Twenty subjects participated in the study. Ten subjects with no history of knee complaints and normal clinical examination were used as controls. The control subjects were aged between 26 and 39 years. There were five females and five male subjects. Ten subjects with 
unilateral PCL injuries were recruited for the study. The subjects were aged between 18 and 47 years. There were five females and five males. Isolated PCL injury was diagnosed on clinical examination and MRI. On clinical examination PCL injury was suggested by posterior sag and posterior draw test. The dial test was used to exclude subjects with concomitant posterolateral corner injuries. Subjects were excluded if there were any contraindications to MRI, may have been pregnant, or if they were over $180 \mathrm{~cm}$ tall (to permit knee flexion in the MRI tunnel). PCL injuries were sustained from a time period of 3 months to 21 years before the study examination. Nine subjects sustained the injury through sports (four from netball, four from rugby and one from soccer) and one subject sustained the injury outside of sport. All subjects provided informed consent. Normal knees were used as controls rather than the healthy contralateral knees because anatomical variations in tibial slope have been identified as risk factors for anterior cruciate ligament injury [13]. Ethics approval for the study was obtained from the Department of ACT Health and Australian National University Human Research Ethics Committees.

\subsection{MRI imaging procedure}

Subjects performed a supine leg press between 0 and $90^{\circ}$ on a wooden frame with a sliding footplate fitted to the MRI couch. The leg press was weighted by a $150 \mathrm{~N}$ load via a rope and pulley to resist leg extension and thereby simulate a weight bearing squat (Fig. 1). Elastic straps stabilised the thighs, feet and ankles. Imaging of both knees simultaneously was performed. Parasagittal images perpendicular to the tibial plateau were generated through each knee.

\subsection{Tibiofemoral contact point measurement}

The position of the tibiofemoral contact (TFC) with the tibial plateau was recorded as the distance from the posterior tibial cortex to the point of the TFC of the medial and lateral femoral condyle (Fig. 2). Where contact occurred over a wide area, the area centroid was used. To account for variation in the size of subjects, cortex to contact distance measurements were normalised to a tibial plateau size of $50 \mathrm{~mm}$. The mean anterior-posterior diameter of the medial tibial plateau was $48 \pm$ $5.4 \mathrm{~mm}$, and the lateral tibial plateau was $41 \pm 2.47 \mathrm{~mm}$.

\subsection{Flexion facet centre measurement}

The position of the flexion facet centre (FFC) over the tibial plateau was located by using a three stage measurement technique with a computer assisted design program (Fig. 3). First, the FFC was identified by fitting a circle to define the flexion arc of the posterior condyle. This involved using an arc function to identify three points on the posterior aspect of the femur which could then be incorporated into a circle of bit fit. Second, the tibial plateau was defined by a line

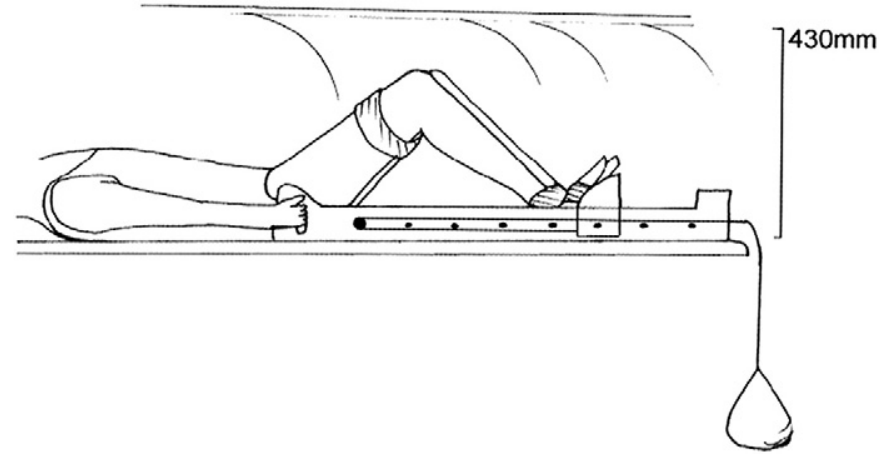

Fig. 1. Subjects' position in the MRI scanning tunnel. The knees were positioned at $15^{\circ}$ intervals between 0 and $90^{\circ}$ flexion, pressing down through the feet against a $150 \mathrm{~N}$ load.

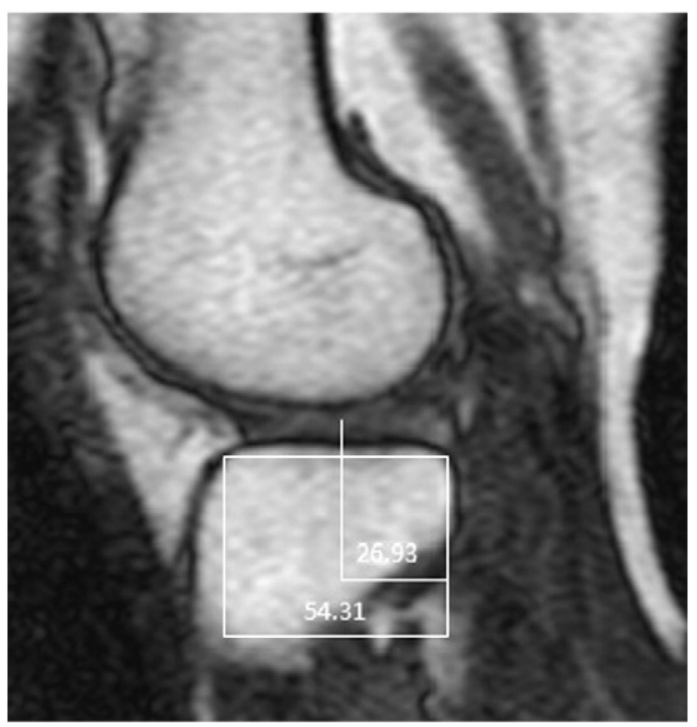

Fig. 2. Sagittal images through the centre of the compartment of the knee were used to measure the tibiofemoral contact (TFC). The distance was measured through the posterior tibial cortex to the centre of the area of contact.

from the posterior tibial cortex, parallel to the tibial plateau. Lastly, a line was drawn through the FFC perpendicular to the tibial plateau line to measure the distance from the posterior tibial cortex to the intersection of the perpendicular line.

\subsection{Precision}

The precision of both methods of measurement was tested by repeating measurement from the original scanned images on two occasions at least $24 \mathrm{~h}$ apart. The precision of mapping the contact points for the medial and lateral compartments was very high with intra class correlation 0.95 (99\% confidence interval was 0.920 .96 ). The precision of measuring of the FFC was also very high with intra class correlation of 0.93 (95\% confidence interval was $0.88-0.93$ ). The greatest difference observed between the repeated measurements was $0.7 \mathrm{~mm}$ for the mapping the TFC point and $0.9 \mathrm{~mm}$ for mapping the FFC.

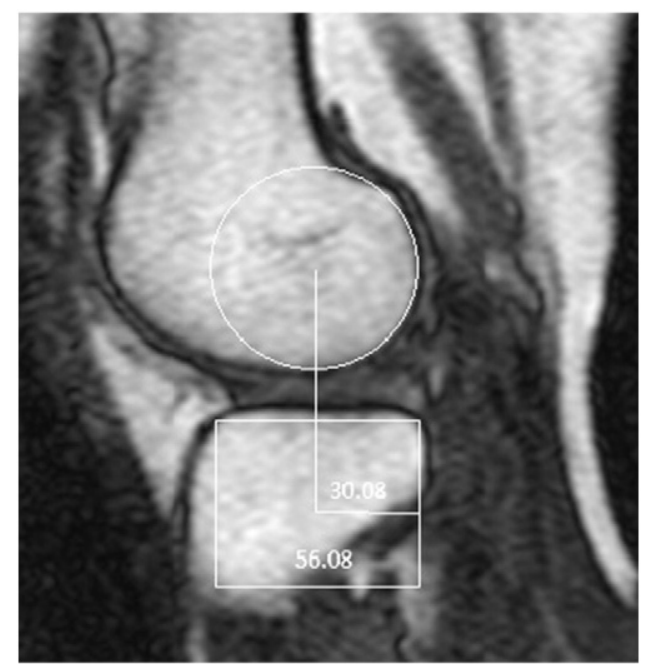

Fig. 3. The position of the flexion facet centre over the tibial plateau was measured in three steps: the arc and centre (FFC) of the posterior femoral condyle were defined, the tibial plateau was defined, and the distance from the perpendicular through the centre to the posterior tibial cortex was determined. 


\subsection{Statistical analysis}

Statistical analysis was carried out using statistiXL version 1.8 for Microsoft Excel. A two-way repeated measures analysis of variance with Tukey and Scheffe post hoc tests were used to compare the tibiofemoral contact points and FFC positions between the healthy, the PCL deficient knees and the contralateral side. A p value of less than 0.05 was regarded as statistically significant.

\section{Results}

Table 1 shows the average and standard deviations for the TFC points and FFC for the healthy and PCL injured knees.

\subsection{Sagittal plane articulation of the tibiofemoral joint in healthy subjects}

3.1.1. Tibiofemoral contact point

In the healthy knee the TFC point moved anterior to posterior as the knee flexed from 0 to $90^{\circ}$ (Fig. 4). In full knee extension the medial compartment had a more anterior mean contact point than the lateral compartment. Between 0 and $30^{\circ}$ the mean contact point in the medial compartment moved posteriorly by $5 \mathrm{~mm}$, which was $0.16 \mathrm{~mm}$ per degree. Between 0 and $30^{\circ}$ the mean contact point in the lateral compartment moved posteriorly by $3 \mathrm{~mm}$, which was $0.10 \mathrm{~mm}$ per degree. Between 45 and $90^{\circ}$ the mean contact point in the medial compartment did not move posteriorly as much, $3 \mathrm{~mm}$ in $45^{\circ}$, or $0.07 \mathrm{~mm}$ per degree. The mean contact point in the lateral compartment also did not move posteriorly as much $4 \mathrm{~mm}$ in $45^{\circ}$, or $0.08 \mathrm{~mm}$ per degree.

\subsubsection{Flexion facet centre}

In the medial compartment the mean FFC was positioned posteriorly over the tibial plateau in knee extension (Fig. 5). The medial mean FFC moved anteriorly by $2 \mathrm{~mm}$ as the knee flexed from 0 to $15^{\circ}$. From 30 to $90^{\circ}$ the medial mean FFC returned to its posterior position over the medial tibial plateau. During the entire flexion arc form 0 to $90^{\circ}$ the medial FFC was positioned over the tibial plateau within a mean distance of $23 \mathrm{~mm}$ to $20 \mathrm{~mm}$ from the posterior tibial cortex. In the lateral compartment the mean FFC moved posteriorly as the knee flexed from 0 to $90^{\circ}$. At full extension the mean FFC was located over the tibial plateau $25 \mathrm{~mm}$ from the posterior tibial cortex. At $90^{\circ}$ the mean FFC in the lateral compartment was located over the tibial plateau $17 \mathrm{~mm}$ form the posterior tibial cortex. The mean FCC in lateral compartment had moved $8 \mathrm{~mm}$ at an average of $0.09 \mathrm{~mm}$ per degree.

3.2. Sagittal plane articulation of the tibiofemoral joint in subjects with an isolated PCL injury

\subsubsection{Tibiofemoral contact point}

In the medial compartment of the PCL injured knee the mean TFC point was located more anteriorly on the tibia throughout the flexion arc from 0 to $90^{\circ}$ compared to the medial compartment of the healthy knee (Fig. 4). In extension the mean TFC point was located $32 \mathrm{~mm}$ from the posterior tibial cortex. From 0 to $30^{\circ}$ the difference in the

Table 1

The position of the tibiofemoral contact point and flexion facet centre in the healthy and PCL injured knees of 20 subjects (mean and standard deviation) measured in millimetres from the posterior tibial cortex.

\begin{tabular}{|c|c|c|c|c|c|c|c|c|}
\hline & \multicolumn{4}{|c|}{ Tibiofemoral contact } & \multicolumn{4}{|c|}{ Flexion facet centres } \\
\hline & \multicolumn{2}{|c|}{ Healthy } & \multicolumn{2}{|c|}{ PCL injured } & \multicolumn{2}{|c|}{ Healthy } & \multicolumn{2}{|c|}{ PCL injured } \\
\hline & Mean & SD & Mean & SD & Mean & SD & Mean & SD \\
\hline \multicolumn{9}{|c|}{ Lateral compartment } \\
\hline 0 & 29 & 2.3 & 30 & 1.0 & 25 & 1.5 & 26 & 1.1 \\
\hline 15 & 27 & 1.8 & 28 & 0.6 & 24 & 1.4 & 24 & 0.7 \\
\hline 30 & 26 & 1.6 & 26 & 1.6 & 22 & 1.6 & 23 & 1.0 \\
\hline 45 & 22 & 1.6 & 22 & 1.1 & 20 & 1.6 & 21 & 0.4 \\
\hline 60 & 19 & 1.8 & 21 & 1.0 & 18 & 1.8 & 19 & 1.4 \\
\hline 75 & 18 & 1.7 & 20 & 0.7 & 17 & 1.7 & 18 & 1.3 \\
\hline 90 & 18 & 1.9 & 19 & 1.3 & 17 & 1.9 & 18 & 1.4 \\
\hline \multicolumn{9}{|c|}{ Medial compartment } \\
\hline 0 & 31 & 1.3 & 32 & 1.6 & 21 & 1.3 & 23 & 1.6 \\
\hline 15 & 29 & 1.2 & 30 & 1.3 & 23 & 1.2 & 22 & 0.7 \\
\hline 30 & 26 & 1.3 & 27 & 1.4 & 22 & 1.3 & 23 & 0.9 \\
\hline 45 & 24 & 1.4 & 27 & 1.4 & 22 & 1.4 & 24 & 1.4 \\
\hline 60 & 23 & 1.4 & 26 & 0.6 & 21 & 1.4 & 23 & 0.9 \\
\hline 75 & 21 & 1.4 & 25 & 1.0 & 20 & 1.4 & 23 & 1.8 \\
\hline 90 & 21 & 1.4 & 26 & 0.9 & 21 & 1.4 & 23 & 1.4 \\
\hline
\end{tabular}

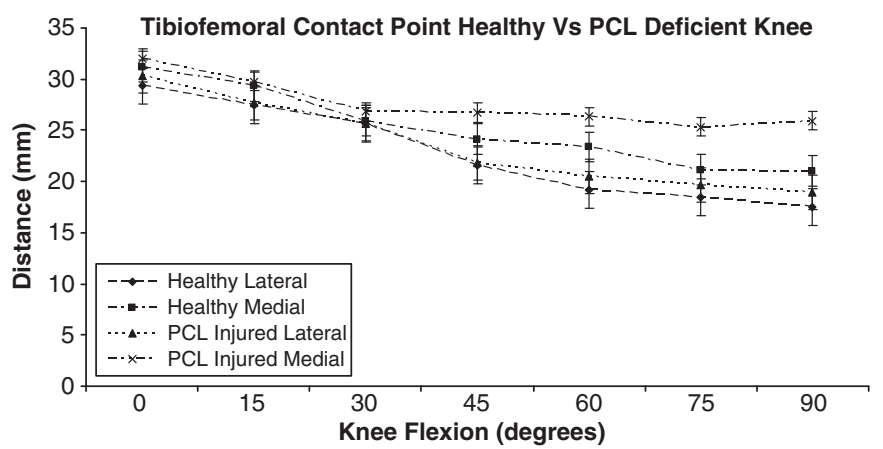

Fig. 4. Graph comparing tibiofemoral contact points in the healthy and PCL injured knee, performing a leg press against a $150 \mathrm{~N}$ load through a flexion arc of 0 to $90^{\circ}$. The pattern of tibiofemoral contact positions in healthy and PCL injured knees is similar in the lateral compartment. The position of the tibiofemoral contact point is significantly more anterior in the medial compartment above $45^{\circ}$ of flexion for the PCL injured group.

location of the contact points between the PCL injured and healthy knees were not statistically significant $(\mathrm{p}>0.05)$. The anterior location of medial compartment mean TFC point in the PCL deficient knee was statistically significant from 45 to $90^{\circ}$ at all measured points $(\mathrm{p}<0.05)$. The greatest difference between the mean TFC points occurred at 75 and $90^{\circ}$, the difference being $4 \mathrm{~mm}$ and $5 \mathrm{~mm}$ respectively. In the lateral compartment the mean TFC point of the PCL injured knees was located more anterior than the mean TFC point of the lateral compartment of the healthy knee throughout the flexion range but there was no statistically significant difference at any of the measured points.

\subsubsection{Flexion facet centre}

In the medial compartment of the PCL injured knee the mean FFC was centred more anteriorly on the tibia compared to the mean FFC of the medial compartment of the healthy knee (Fig. 5). This was not significant at 0,15 and $30^{\circ}$ but statistically significant at $45,60,75$ and $90^{\circ}$. The greatest difference of the FFC occurred at $75^{\circ}$ of flexion, which was $3 \mathrm{~mm}$. In the lateral compartment of the PCL deficient knee the FFC was centred more anteriorly than the healthy knee over the tibial plateau as the knee flexed through 0 to $90^{\circ}$, however, there was no statistical difference.

3.3. Sagittal plane articulation of the tibiofemoral joint of the healthy contralateral knee in subjects with an isolated PCL injury

There was no significant difference in the position of both the mean TFC point and mean FFC between the contralateral healthy knee and normal knee in both the medial and lateral compartments throughout the flexion arc from 0 to $90^{\circ}$. In the medial compartment the mean TFC and FFC positions were significantly more anterior in the PCL injured knee compared with the contralateral side with the greatest difference replicating the relationship between the normal and PCL deficient knee. Similarly, there was no difference in the lateral compartment in the positions of the mean TFC point and FFC between the PCL deficient knee and the contralateral side.

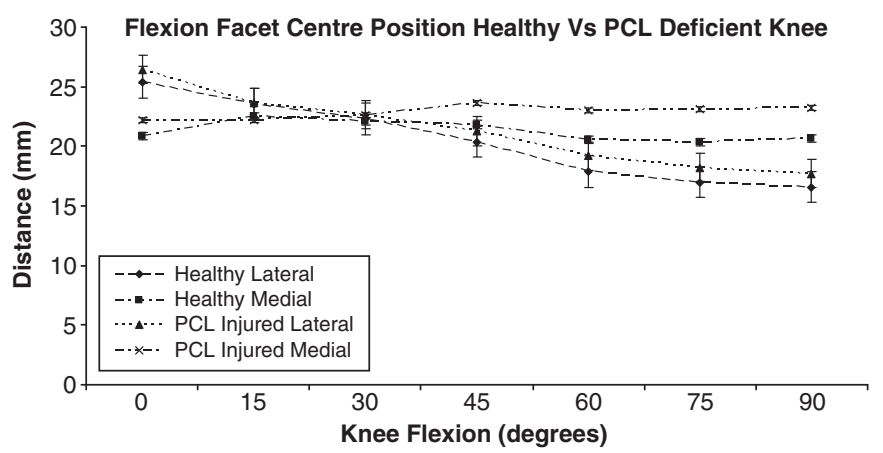

Fig. 5. Graph comparing flexion facet centre position in the healthy and PCL injured knee, performing a leg press against a $150 \mathrm{~N}$ load through a flexion arc of 0 to $90^{\circ}$. The position of the flexion facet centre in healthy and PCL injured knees is similar in the lateral compartment. The position of the flexion facet centre is significantly more anterior in the medial compartment above $45^{\circ}$ of flexion for the PCL injured group. 


\section{Discussion}

The aim of this study was to investigate the effect of an isolated PCL injury on the sagittal plane articulation of the tibiofemoral joint. This study demonstrated that PCL deficiency produced a significant change in the sagittal plane articulation of the tibiofemoral joint in the medial compartment between 45 and $90^{\circ}$ of knee flexion compared to the healthy knee and contralateral side. Specifically, the TFC point and FFC move more anteriorly as the knee flexes. Further, this study demonstrated that PCL deficiency produced no significant change in the sagittal plane articulation of the lateral compartment of the tibiofemoral joint. The results are consistent with cadaveric studies which show that the PCL is an important constraint to posterior tibial subluxation and clinical studies which show that chronic PCL deficient knees are associated with medial compartment chondral lesions.

This study used an in vivo model to describe the sagittal plane articulation of the PCL deficient knee. The study incorporated a supine leg press against a $150 \mathrm{~N}$ load through a flexion arc of 0 to $90^{\circ}$ to simulate a squat. The benefits of using this invivo model over cadaveric sectioning studies is that this model allows the study to replicate the neuromuscular contribution to joint stability that occurs in the clinical setting of PCL injury. This study used healthy knees as the control rather than the contralateral knee as there have been studies that have shown that variations in bony and soft tissue anatomy may predispose to ligamentous injuries of the knee [14-16]. However, the results of this study showed there was no difference in the articulation pattern of the healthy and contralateral knees.

The limitations of this study include supine analysis and small sample size. The supine leg press was intended to simulate a squat. It is difficult to extrapolate whether this replicates the forces during sporting or activities of daily living and as such could potentially be a source of error in our results. Although the number in the study was small (10 healthy knees and 10 PCL injured knees), the number was sufficient to demonstrate a significant difference in the sagittal plane articulation of the PCL deficient knee. Furthermore, in vivo studies on PCL kinematics have used similar or smaller participant numbers [17].

The findings in this study are consistent with results of several cadaveric studies in the literature. Cadaveric studies have shown that isolated cutting of the PCL allows a minimal increase in posterior draw when the knee is extended, and that there is a greater increase in laxity as the knee flexes with the greatest displacement at $90^{\circ}$ of knee flexion [18-23]. Veltri et al. demonstrated that the posterolateral structures of the knee exert a maximal posterior tibial restraining force from 0 to $30^{\circ}$ of knee flexion [24]. Intact posterolateral structures explain why anterior tibial displacement of the PCL injured knee in our study was not significant between 0 and $30^{\circ}$ of knee flexion. In addition, Robinson et al. analysed the role of the posteromedial capsule in resisting posterior translation of the tibia. They demonstrated that the posteromedial capsule controlled valgus, internal rotation, and posterior drawer in extension, resisting $42 \%$ of a $150-\mathrm{N}$ drawer force when the tibia was in internal rotation. Their finding may also explain why PCL injury did not produce significant anterior displacement of the tibia at less than $30^{\circ}$ of knee flexion in our study. However, there have been no cadaveric studies that have assessed the relative contributions of the medial and lateral compartments of the knee to posterior translation.

There have been very few in vivo studies investigating the sagittal plane articulation of the PCL deficient knee. Logan et al. analysed the FFC in 6 subjects with isolated PCL rupture undergoing a weight bearing squat using open access MRI [17]. They reported significant posterior subluxation of the medial tibia at $0,20,45$ and $90^{\circ}$ of knee flexion. In addition, they reported that the sagittal plane articulation of the lateral compartment was not altered by posterior cruciate ligament rupture. Our results are in agreement with Logan et al. which demonstrate altered articulation in the medial compartment. One point of difference is that Logan et al. noted significant altered articulation throughout the arc of flexion from to 0 to $90^{\circ}$ whereas we found that articulation was significantly different from 45 to $90^{\circ}$. There are two possible explanations for this discrepancy. Firstly, it is not clear whether Logan et al. excluded subjects with concomitant posterolateral corner injury as cadaveric studies have shown that posterolateral corner injuries are most important constraint to posterior tibial translation from 0 to $30^{\circ}$ [24]. The weight bearing model of Logan et al. is a more functional model of testing the kinematics of PCL deficiency than our model in which the subject is supine. The increased forces with weight bearing may accentuate posterior tibial subluxation in the PCL deficient knee. It is also important to note that in our study we used two methods, the TFC point and FFC, to assess knee kinematics whereas Logan et al. had only used the FFC. Both methods in our study had concurring findings. Castle et al. used stress radiography to assess the effects of PCL rupture on knee articulation at 35 and $70^{\circ}$ of flexion [25]. They showed that at $70^{\circ}$ of flexion the tibia is subluxed posteriorly by $6 \mathrm{~mm}$ and no difference at $35^{\circ}$ of flexion. They did not analyse the relative contributions of the medial and lateral sides of the knee to posterior tibial translation but their results are nevertheless in agreement with our study. Donell et al. published on a pilot study that compared tibiofemoral articulation of five PCL injured subjects with four normal subjects who underwent MRI with a novel splint that stresses the tibia in a posterior direction [26]. They reported that there was no marked posterior tibial translation for the PCL injured knee in posterior drawer compared to the normal knee. Possible reasons why their findings may differ from our study are the effects of secondary constraint from the posterolateral structures, the increased force on the tibia with a supine leg press compared to a static draw and the small number and therefore power of their study. Unfortunately, their follow up study only reported on ACL, not PCL rupture [27].

The findings of our study complement those of arthroscopic studies that have shown that chronic rupture of the PCL leads to increased incidence of chondral lesions on the medial femoral condyle. Strobel et al. arthroscopically evaluated subjects with a more than 5 year history of a symptomatic PCL injury. They reported that $80 \%$ of subjects had an articular lesion of the medial femoral condyle and $50 \%$ had articular lesions at the patellofemoral articulation [8]. These findings complement those of our study which show that medial compartment is site of abnormal articulation in the PCL deficient knee. Cadaveric studies have complemented these findings by showing that isolated PCL sectioning increases the articular contact pressure within the medial compartment of the knee [28].

In conclusion, there is significant posterior subluxation of the medial tibial plateau form 45 to $90^{\circ}$ in the PCL deficient knee. Physiotherapy and operative interventions should therefore aim to address the altered sagittal plane articulation in the medial compartment of the PCL deficient knee.

\section{Conflict of interest}

None.

\section{Acknowledgment}

The authors would like to acknowledge support from the radiology department of Canberra Hospital in completing this study.

\section{References}

[1] Race A, Amis AA. The mechanical properties of the two bundles of the human posterior cruciate ligament. J Biomech 1994;27:13-24.

[2] Race A, Amis AA. Loading of the two bundles of the posterior cruciate ligament: an analysis of bundle function in a-P drawer. J Biomech 1996;29:873-9.

[3] Amis AA, Gupte CM, Bull AM, Edwards A. Anatomy of the posterior cruciate ligament and the meniscofemoral ligaments. Knee Surg Sports Traumatol Arthrosc 2006;14:257-63. 
[4] Andrews JR, Edwards JC, Satterwhite YE. Isolated posterior cruciate ligament injuries. History, mechanism of injury, physical findings, and ancillary tests. Clin Sports Med 1994;13:519-30.

[5] Colvin AC, Meislin RJ. Posterior cruciate ligament injuries in the athlete: diagnosis and treatment. Bull NYU Hosp Jt Dis 2009;67:45-51.

[6] Fanelli GC, Giannotti BF, Edson CJ. The posterior cruciate ligament arthroscopic evaluation and treatment. Arthroscopy 1994;10:673-88.

[7] Jordan SS, Campbell RB, Sekiya JK. Posterior cruciate ligament reconstruction using a new arthroscopic tibial inlay double-bundle technique. Sports Med Arthrosc 2007; 15:176-83.

[8] Strobel M], Weiler A, Schulz MS, Russe K, Eichhorn HJ. Arthroscopic evaluation of articular cartilage lesions in posterior-cruciate-ligament-deficient knees. Arthroscopy 2003; $19: 262-8$

[9] Scarvell JM, Smith PN, Refshauge KM, Galloway H, Woods K. Comparison of kinematics in the healthy and ACL injured knee using MRI. J Biomech 2005;38: 255-62.

[10] Scarvell JM, Smith PN, Refshauge KM, Galloway HR, Woods KR. Comparison of kinematic analysis by mapping tibiofemoral contact with movement of the femoral condylar centres in healthy and anterior cruciate ligament injured knees. J Orthop Res 2004;22:955-62.

[11] Scarvell JM, Smith PN, Refshauge KM, Galloway HR, Woods KR. Evaluation of a method to map tibiofemoral contact points in the normal knee using MRI. J Orthop Res 2004;22:788-93.

[12] Scarvell JM, Smith PN, Refshauge KM, Galloway HR, Woods KR. Association between abnormal kinematics and degenerative change in knees of people with chronic anterior cruciate ligament deficiency: a magnetic resonance imaging study. Aust J Physiother 2005;51:233-40.

[13] Sward P, Kostogiannis I, Roos H. Risk factors for a contralateral anterior cruciate ligament injury. Knee Surg Sports Traumatol Arthrosc 2010;18:277-91.

[14] Defrate LE, Papannagari R, Gill TJ, Moses JM, Pathare NP, Li G. The 6 degrees of freedom kinematics of the knee after anterior cruciate ligament deficiency: an in vivo imaging analysis. Am J Sports Med 2006;34:1240-6.

[15] Li G, Papannagari R, Li M, Bingam J, Nha KW, Allred D, et al. Effect of posterior cruciate ligament deficiency on in vivo translation and rotation of the knee during weightbearing flexion. Am J Sports Med 2008;36:474-9.

[16] Papannagari R, Gill TJ, Defrate LE, Moses JM, Petruska AJ, Li G In vivo kinematics of the knee after anterior cruciate ligament reconstruction: a clinical and functional evaluation. Am J Sports Med 2006;34:2006-12.
[17] Logan M, Williams A, Lavelle J, Gedroyc W, Freeman M. The effect of posterior cruciate ligament deficiency on knee kinematics. Am J Sports Med 2004;32:1915-22.

[18] de Abreu MR, Kim HJ, Chung CB, Jesus JM, Cho J, Tudell D, et al. Posterior cruciate ligament recess and normal posterior capsular insertional anatomy: MR imaging of cadaveric knees. Radiology 2005;236:968-73.

[19] Fox RJ, Harner CD, Sakane M, Carlin GJ, Woo SL. Determination of the in situ forces in the human posterior cruciate ligament using robotic technology. A cadaveric study. Am J Sports Med 1998;26:395-401.

[20] Hagemeister N, Duval N, Yahia L, Krudwig W, Witzel U, de Guise JA. Computer based method for the three-dimensional kinematic analysis of combined posterior cruciate ligament and postero-lateral complex reconstructions on cadaver knees. Knee 2003;10:249-56.

[21] Hoher J, Vogrin TM, Woo SL, Carlin GJ, Aroen A, Harner CD. In situ forces in the human posterior cruciate ligament in response to muscle loads: a cadaveric study. J Orthop Res 1999;17:763-8.

[22] Rosenberger RE, Bale RJ, Kneisl C, Krappinger D, Knoflach M, Attal R. Twodimensional fluoroscopic navigation in posterior cruciate ligament reconstruction: a preclinical cadaver study. Arch Orthop Trauma Surg 2010; Aug;130(8): 971-6 [Electronic publication ahead of print 2010 Jan 5].

[23] Wang CJ, Chen HH, Chen HS, Huang TW. Effects of knee position, graft tension, and mode of fixation in posterior cruciate ligament reconstruction: a cadaveric knee study. Arthroscopy 2002;18:496-501.

[24] Veltri DM, Deng XH, Torzilli PA, Warren RF, Maynard MJ. The role of the cruciate and posterolateral ligaments in stability of the knee. A biomechanical study. Am J Sports Med 1995;23:436-43.

[25] Castle Jr TH, Noyes FR, Grood ES. Posterior tibial subluxation of the posterior cruciate-deficient knee. Clin Orthop Relat Res 1992:193-202.

[26] Donell ST, Marshall TJ, Darrah C, Shepstone L. Cruciate ligament assessment in MRI scans: a pilot study of a static drawer technique. Knee 2006;13:137-44

[27] Al-Dadah O, Shepstone L, Marshall TJ, Donell ST. Secondary signs on static stress MRI in anterior cruciate ligament rupture. Knee 2010; Sep 3 [Electronic publication ahead of print].

[28] Ahmed AM, Burke DL, Yu A. In-vitro measurement of static pressure distribution in synovial joints - Part II: Retropatellar surface. J Biomech Eng 1983;105:226-36. 